\title{
Application of Iced Normal Saline Combined with Cocktail Perfusion in Total Knee Arthroplasty: randomized controlled trial
}

\section{Laijian Sui}

Yuhuangding Hospital

\section{Xiufeng Wang}

Zhaoyuan Renmin Hospital

\section{Pengzhou Gai}

Yuhuangding Hospital

Jinwei Wang

Yuhuangding Hospital

\section{Xiaojun Qi}

Yuhuangding Hospital

Jing Wang

Yuhuangding Hospital

\section{Aihua Jiang}

Yuhuangding Hospital

Guangda Wang ( $\nabla$ wgdsh123@sohu.com )

Yuhuangding Hospital

\section{Research Article}

Keywords: cocktail therapy, iced normal saline, total knee arthroplasty (TKA), postoperative recovery

Posted Date: October 18th, 2022

DOI: https://doi.org/10.21203/rs.3.rs-1080029/v2

License: (1) (1) This work is licensed under a Creative Commons Attribution 4.0 International License. Read Full License 


\section{Abstract \\ Objective:}

The present study was designed to investigate the safety and effectiveness of iced normal saline combined with cocktail perfusion during total knee arthroplasty (TKA). This was a random, double-blind, parallel-group study conducted in China.

\section{Methods.}

Seventy patients undergoing unilateral total knee replacements were assessed in the present study. Among them, sixty patients with confirmed primary knee osteoarthritis in stage IV were recruited and divided into three groups randomly, three different intro-operative articular cavity perfusion treatments were given according to the randomized and controlled rule. One way ANOVA analysis on visual analogue scale (VAS) score, functional recovery, drainage, and edema of the affected limb were performed to assess the efficiency of the treatment in the following three days after the operation. The participants, care givers, and those assessing the outcomes were blinded to group assignment.

\section{Results.}

Postoperative drainage in group $A(n=20)$ and $B(n=20)$ reduced significantly $(P<0.05)$. The IHC scores of the surgical limbs were markedly lower comparing with the control group $(n=20)(P<0.05)$. No differential postoperative edema was observed and the patient acquired better rehabilitation in group $A$ and $B$ than control group. Compared with group $A$, no differential postoperative drainage, edema or other side effects were seen in group $B(P>0.05)$. The VAS score of group $B$ was significantly lower than in group $A(P<0.05)$. Postoperative recovery of the surgical limb function in group $B$ was better than in group $A(P<0.05)$.

\section{Conclusions:}

Intra-operation articular cavity perfusion therapy with iced normal saline combined with cocktail perfusion therapy can greatly reduce the early inflammation, contributing to the better rehabilitation of TKA.

\section{Background}

Knee osteoarthritis (KOA) is a common disease caused by the degradation of articular cartilage, which is manifested as painful, edema, stiffness and restrained mobility of the knee. Although KOA is not fatal, it seriously reduces the life quality of the elder patients. According to the survey in the USA, females with KOA suffered higher risks than the normal, which might be correlated with the disordered mental health 
induced by the chronic pain and the sports barrier. Meanwhile, the morbidity of obesity and cardiovascular event increased due to the limited exercise caused by the KOA which severely impact the quality of the human life.

Early symptoms of KOA could be relieved by the conserve therapy such as health education, sports therapy and drug treatment. Clinical intervene including arthroscopic debridement and cartilage graft were also applied to retard the progression of KOA. However, advanced KOA was irreversible. Total knee arthroplasty, which was acknowledged as the most effective treatment for advanced knee osteoarthritis, was widely accepted to relieve the pain of KOA and restore the function of the knee. Nevertheless, many patients refused TKA due to the fear of the pain post-operation.

Recent year, how to alleviate postoperative pain and promote rapid recovery of patients has witnessed an increased research interest. In the present study, application of iced normal saline combined cocktail perfusion therapy was tested to decrease the post-operative pain of the surgical limb and restore the limb function as soon as possible.

\section{Methods}

\section{Trial design, participants, and study settings:}

Seventy patients undergoing unilateral total knee replacement in joint surgery of Yantai Yuhuangding Hospital from 2nd March 2018 to 1st June 2018 were assessed in the present study (IRB number: 2018149 Human Research Ethics Committee of Yuhuangding Hospital supplemental file 1). Sixty patients with confirmed primary knee osteoarthritis in stage IV by preoperative X-ray were included in present study, whilst subsequent arthritis (including rheumatoid arthritis, Gouty arthritis, traumatic arthritis, etc.) were excluded. Kellgren-Lawrence Radiology standards for stage IV were listed as follows: obviously narrowed joint space; vast osteophytes are formed; severe osteosclerosis under the cartilage; bone hypertrophy and evident deformity (Fig. 1). After assessment, sixty qualified patients who voluntarily participating in this study have understood the research project were included in the present study and the corresponding consent forms were signed. Patients were randomly classified into three groups with different interventions, each group contains 20 patients. 20 patients with routine treatment (only with intra-articular infusion of $20 \mathrm{ml}$ tranexamic acid) were recorded as the control group (Fig. 3). All the study personnel including the patients, their family members, the nursing staff and the leading operator were blind to the treatment allocation.

\section{Interventions}

Systemic preoperative inspections were performed to exclude any contraindications to surgery, and the consent forms were signed before operation.

Recruited patients were divided into three groups with different recipes. Group A: twenty patients who were given intraoperative joint cavity infusion therapy using $20 \mathrm{ml}$ tranexamic and $40 \mathrm{ml}$ iced normal 
saline; Group B twenty patients who were given $20 \mathrm{ml}$ of iced normal saline, $20 \mathrm{ml}$ of iced cocktail and $20 \mathrm{ml}$ of tranexamic; the left twenty patients were given joint cavity infusion therapy with $20 \mathrm{ml}$ tranexamic only as the control group. Intravertebral anaesthesia was selected for the surgery and the operations were performed by the same surgical team. The average operation time was about 1.5 hours, which lasted from the compression of the tourniquet to completed wound dressing.

Intraoperative cocktail infiltration anaesthesia and adductor tube blocking therapy were performed routinely for all the patients. Posterior stable Prothesis (Nexgen-LPS, Zimmer, US) was applied for the operation (Fig. 2). Cocktail was formulated during the operation by mixing Ropivacaine 150mg, Morphine $10 \mathrm{mg}$ and Epinephrine $1 \mathrm{mg}$, then normal saline was used to dilute the mixture to $60 \mathrm{ml}$. Ropivacaine 150mg was used for the block therapy. High pressure drainage system (PFM, Germany) was applied intraoperation and the drainage tube was clamped for 6 hours after operation. The drainage tube was removed 24 hours after the operation. The first dose of anticoagulant (nadroparin calcium 4100 IU) was applied 8 hours after the operation. Tranexamic acid was also applied during the perioperative period both cavity irrigation and intravenous infusion. The first dose for intravenous infusion was performed after loosening the tourniquet, repeated doses of tranexamic acid were given intravenously immediately when the patients were sent back to the ward and every 3 hours for 3 times. Postoperative rehabilitation training was performed routinely after the operation as soon as possible.

\section{Outcomes}

The visual analogue scale (VAS) scoring system was applied to evaluate the pain in the following 24,48 and 72 hours after surgery. The hospital for special surgery (HSS) scores of the following three days after surgery were assessed. The 24 -hour postoperative drainage volume and the limb swelling values were recorded.

Based on the analysis of subjective feelings and objective data such as the drainage, the swelling value, etc, of patients after surgery, the effect of intra-operative iced saline combined cocktail perfusion therapy was evaluated.

\section{Sample size}

To investigate the reduction of the post-operative pain and recovery of the surgical limb function, a sample size of 20 patients per group was necessary. To recruit this number of patients a 3-month period was anticipated.

\section{Randomisation:}

For allocation of the participants, a computer-generated list of random numbers was used, participants were randomly assigned following simple randomization.

\section{Statistical analyses}

Comparison between the two groups is completed by student T-test whilst one way ANOVA was used to compare the difference among the three groups. $P<0.05$ was regarded as statistical difference. One way 
ANOVA and student T-test were performed using SPSS 26. 0.

\section{Results}

Participant flow and recruitment

For each group, twenty participants who were randomly assigned, received intended treatment, and were analysed for the primary outcome (Fig. 3).

Age-eligible participants were recruited from 2nd March 2018 to 1st June 2018. Participants undergoing unilateral total knee replacement in joint surgery of Yantai Yuhuangding Hospital during these 3 months period.

\section{General conditions of patients in each group}

No significant difference was observed in gender, age, pre-operative HSS scores and pre-operative joint circumferences among three groups. The samples were qualified for the requirement of homogeneity. (Table 1)

Table 1

Comparison of the general conditions (Mean \pm SD)

\begin{tabular}{|llllll|}
\hline & Age & Gender & $\begin{array}{l}\text { Preoperative HSS } \\
\text { scores }\end{array}$ & $\begin{array}{l}\text { Pre-operative joint } \\
\text { circumferences }(\mathbf{c m})\end{array}$ \\
\cline { 3 - 5 } & & Female & Male & & \\
\hline $\begin{array}{l}\text { Control } \\
\text { group }\end{array}$ & $\begin{array}{l}66.55 \pm \\
5.58\end{array}$ & $\mathrm{n}=13$ & $\mathrm{n}=7$ & $45.05 \pm 3.19$ & $46.57 \pm 3.22$ \\
\hline Group A & $\begin{array}{l}65.75 \pm \\
4.77\end{array}$ & $\mathrm{n}=14$ & $\mathrm{n}=6$ & $44.65 \pm 2.99$ & $46.73 \pm 3.06$ \\
\hline Group B & $\begin{array}{l}65.70 \pm \\
4.92\end{array}$ & $\mathrm{n}=12$ & $\mathrm{n}=8$ & $45.45 \pm 3.08$ & $45.82 \pm 3.58$ \\
\hline
\end{tabular}

Statistical analysis of postoperative observation indexes of the three groups.

One way ANOVA was performed to compare the differences of the 24-hour

postoperative drainage volume, postoperative pain score $(24,48,72 \mathrm{~h})$ and HSS score on the third day after the operation among the three groups. No differential swelling value was observed at 24 hours after operation $(P>0.05)$. Statistically altered postoperative drainage ( 24 hours after operation) and postoperative pain score $(24,48,72 \mathrm{~h}$ after operation) and HSS score on the third day after surgery were shown in both group $A$ and group B, comparing with the control group (Table 2). Compared with group A, there was no significant difference in group B on HSS score at 24 hours and the third day after surgery, whilst postoperative pain scores of group B present significant difference $(P<0.01)$. Details are shown in Table 2 and Fig. 4. 
Table 2

Statistical analyses of the postoperative indexes (Mean \pm SD).

\begin{tabular}{|c|c|c|c|c|c|c|}
\hline & \multirow[t]{2}{*}{ Drainage(ml) } & \multirow{2}{*}{$\begin{array}{l}\text { Swelling } \\
\text { value(cm) }\end{array}$} & \multicolumn{3}{|c|}{ Postoperative VAS scores } & \multirow{2}{*}{$\begin{array}{l}\text { Postoperative } \\
\text { HSS scores }\end{array}$} \\
\hline & & & $24 \mathrm{~h}$ & $48 \mathrm{~h}$ & $72 \mathrm{~h}$ & \\
\hline $\begin{array}{l}\text { Control } \\
\text { group }\end{array}$ & $301.5 \pm 37.1$ & $1.15 \pm 0.67$ & $\begin{array}{l}6.6 \pm \\
0.96\end{array}$ & $\begin{array}{l}5.15 \pm \\
0.96\end{array}$ & $\begin{array}{l}3.7 \pm \\
0.71\end{array}$ & $56 \pm 6.35$ \\
\hline Group A & $\begin{array}{l}140.25 \pm \\
34.4^{\star \star}\end{array}$ & $1.2 \pm 0.53$ & $\begin{array}{l}5.05 \pm \\
0.74^{\star \star}\end{array}$ & $\begin{array}{l}3.50 \pm \\
0.87^{\star \star \star}\end{array}$ & $\begin{array}{l}2.45 \pm \\
0.67^{\star \star \star}\end{array}$ & $61.25 \pm 4.56^{\star \star}$ \\
\hline Group B & $\begin{array}{l}127.5 \pm \\
29.8^{\star *}\end{array}$ & $1.10 \pm 0.60$ & $\begin{array}{l}3.75 \pm \\
0.70^{\star \star \star}\end{array}$ & $\begin{array}{l}2.35 \pm \\
0.873^{\star *}\end{array}$ & $\begin{array}{l}1.55 \pm \\
0.59 * \star\end{array}$ & $61.85 \pm 3.47^{\star \star}$ \\
\hline
\end{tabular}

\section{Postoperative complications}

None of the three groups had serious complications such as infection, poor incision healing, joint dislocation, and vein thrombosis event (VTE). In the control group, 5 patients were given flurbiprofen sodium intravenous pump for pain relief after the operation. No analgesia pump was applied in the experimental group A and the experimental group B; Three patients in the experimental group B suffered from nausea and vomiting after the operation. Luckily the symptoms were not serious, no special treatment was given; None of the three groups of patients received blood products after operation.

\section{Discussion}

\section{Limitations:}

Result from the subjective assessment of patients' feeling and inadequate sample size are limitations of our study. Besides the objective determination from the drainage, edama, if the inflammation factors from the drainage could also be analysed, the assessment would be more convincing.

Generalisability and interpretation:

As the aging of the population and the increasing requirement of the life quality of the elders, total knee arthroplasty, the most effective method to cure advanced KOA, is urging these years. However, postoperative pain and bleeding are still the head-paining issue for both the patients and the arthrologists. Satisfied pain-relieving intervene and bleeding-reduced treatment play pivotal role in acquiring an ideal rehabilitation [1].

Postoperative pain is a nightmare for patients. Studies showed that about $30 \%$ of patients suffered from moderate pain after TKA, and about $60 \%$ of patients reached severe pain [2]. According to Dorr's survey [3], only less than half of the early pain after TKA can be effectively controlled. Severe postoperative pain will exert inverse effect on the cardiovascular, endocrine, respiratory and other systems, as well as the 
internal environment balance, which may also trigger the psychological disorders, such as anxious or insomnia after the operation. In particular, the pain will induce the muscular tension of the knee, which may bring out the resistance for early activity and functional exercise, leading to the unsatisfied rehabilitation. Reuben et al. [4] also believe that uncontrolled early pain after TKA is one of the main causes of postoperative chronic pain.

The pain after TKA is mainly manifested as moderate to severe pain, which is caused by the mechanical damage of surgical dissection. As the constant stimulation of massive pain mediator, lower pain threshold is accumulated for the peripheral nerve pain receptors, meanwhile the stimulation is conducted through nerves to the central nervous system which prolonged the central response time, leading to the increased intensity and eventually a hypersensitivity response.

Effective postoperative analgesia not only alleviates the patient's subjective feelings of pain, but also reduces the post-operative complications such as deep vein thrombosis and pulmonary embolism in the lower limbs caused by lack of exercise. Research revealed that timely active exercise is the most effective method to prevent the adverse thrombosis event, nevertheless, the prerequisite for effective functional exercise after surgery is to obtain the satisfied analgesia. What's more, early active exercise is essential for the recovery of knee function [5]. In addition, the post-operative pain is also closely correlated with patients' age, gender, type of operation, preoperative pain levels (assessed by the HSS scores) and operation time [6]. No statistical difference was observed in the listed influencing factors in present study.

Massive bleeding, including obvious bleeding and invisible bleeding, often occurs after TKA. Bleeding is highly correlated with gender, coagulation function, intraoperative application of tourniquet, prosthesis design, surgical technique, and the use of haemostatic drugs [7]. Obvious bleeding is mainly reflected in the postoperative drainage, whilst postoperative swelling of the soft tissue around the knee joint is the main reflection of invisible bleeding. According to Pattison's [8] research, invisible bleeding is mainly caused by haemolysis and bleeding into the soft tissue space. Excessive bleeding after surgery often results in a reduced haemoglobin, which leads to anaemia and delays early rehabilitation. Anaemia can be corrected by allogeneic blood transfusion in a short time, however, many complications of the blood transfusion, such as the transmission of infectious diseases, side effect of blood transfusion and immunosuppression may follow [9]. Meanwhile, the shortage of blood reserves in recent years has made it more and more difficult to transfuse allogeneic blood. Therefore, effective methods to reduce perioperative pain and postoperative bleeding is essential. Intraoperative joint perfusion with iced normal saline and cocktail is undoubtedly a simple and easily performed method.

Nowadays, cold compress therapy is employed to treat various acute injuries [10,11]. Cold compress can dispel the inflammation mediators by constricting capillaries to intercept the progression of inflammation, contributing to the haemostatic and analgesic effects. Research in acute soft tissue injury revealed that pressurized cold compress treatment can significantly slow down the speed of nerve conduction and reduce the sensitivity of local nerve endings to relieve the pain [12]. The present study 
showed that the VAS scores of patients in the ice-salt water group and ice-salt-salt-mixed cocktail group were significantly lower than those of the control group at $24-, 48-$, and 72 -hours post-operation $(P<0.05)$.

Cocktail therapy in TKA has been clinically applied widely, although no universal criteria is established for the recipe. We modified the cocktail recipe basing on the consensus of experts. In addition to ropivacaine and epinephrine, morphine and triamcinolone acetonide were also formulated and diluted with normal saline to $60 \mathrm{ml}$. Intraoperative extensive injection of cocktails could directly block the signalling transduction of vast pain receptors in the knee joint cavity to achieve a good analgesic effect [13]. Generally, ropivacaine, lidocaine and bupivacaine are used for local anaesthesia. Due to the advantage of long action time, strong anaesthetic intensity and small cardiovascular side effects, ropivacaine was selected in our cocktail. On the other hand, ropivacaine presents exert little effect in blocking the motor nerves, and it does not delay the early postoperative rehabilitation. Although it is a central opioid analgesic, morphine also has periphery receptors. The topical application not only presents a good analgesic effect, but also can avoid the side effect of morphine such as urine storage, lethargy, and dry stool to a certain extent. Adrenaline can constrict local blood vessels, delay the absorption and metabolism of the cocktail, and extend the action time of the cocktail.

Through clinical assessment, the analgesic effect of iced normal saline combined cocktail is much better than just using pure iced normal saline $(P<0.05)$. Thus, the postoperative pain and the inflammation are significantly reduced. As a result, patients can rehabilitate earlier, contributing to the promotes the recovery of knee function. The postoperative HSS scores of patients on the third day after surgery in the iced normal saline group and the iced normal saline combined with cocktail group were markedly higher than that of the control group $(P<0.05)$.

Routinely intra-operative extensive soft tissue loosening, osteotomy and patella repair in TKA lead to enlarged wounds and serious bleeding. How to reduce post-operative bleeding, reduce the incidence of blood transfusion and related postoperative complications is the special attention should be paid after TKA. Previous report showed that cold compresses after TKA proved to significantly lower pain, reduce bleeding and improve the sleep quality [14]. For the experienced surgeon, iced normal saline irrigation could be selected as the easiest and reachable method to tackle with the intraoperative capillaries oozed wound bleeding. In the present study, promoted capillary constriction induced by the iced perfusion in both group $A$ and group $B$ not only decreased the bleeding but also prolonged the action time of local anaesthetics, that is why a better analgesic effect and rehabilitation were observed compared with the control group. On the other hand, the increased local pressure of the joint cavity after the perfusion of ice normal saline could compress the capillaries in the wound, thereby achieving the haemostasis and less drainage. Iced perfusion contributes to the reduced congestion due to the vascular constriction meanwhile, which also relieves the pressure stimulated pain to the sensation. While comparing the rehabilitation between group $A$ and group $B$, the latter group presented better analgesia, which could be accounted as that application of the cocktail within the safe dose significantly suppressed the inflammation in the early postoperative stage of TKA. 
Elastic bandage dressing was also applied in our clinical practice to reduce the leakage of the broken ends of the lymphatic vessels in the joint cavity and promote the venous blood return. Recent years, tranexamic acid is proved to exert pivotal role in controlling hyper fibrinolytic bleeding after TKA, intraoperative and postoperative application of tranexamic acid significantly reduced the incidence of blood transfusion and the postoperative inflammation [15]. In the present study, drainage in the iced normal saline group and the iced normal saline mixed with cocktail group was significantly lower than that in the control group at 24 hours after surgery $(P<0.05)$. Considering the risks of normal saline penetrating into the soft tissue around the joints to induce the joint swelling, we measure the circumference of the thigh $10 \mathrm{~cm}$ above the knee after TKA. No statistical differential circumstance was observed between the joint cavity perfused and control group, which indicated that the perfusion therapy did not increase the postoperative swelling.

\section{Conclusions}

Iced normal saline combined with cocktail infusion during TKA significantly reduced the perioperative blood loss and lower postoperative pain score without increasing the swelling of the surrounding soft tissues, which tended to be a safe and effective clinical practise in TKA. However, due to the limited sample numbers and lack of long-term follow-up study, potential side effects and the more details on this method such as the individually designed perfusion pressure still need further exploration.

\section{List Of Abbreviations}

TKA: total knee arthroplasty

KOA: knee osteoarthritis

VAS: visual analogue scale

HSS: hospital for special surgery

VTE: vein thrombosis event

\section{Declarations}

\section{Registration:}

The present study was retrospectively registered on ClinicalTrials. Gov with the identifierNCT05204056 $(27 / 11 / 2021)$.

\section{Funding:}

This study was supported by Natural Science Foundation of Shandong Province (ZR2017MH075). 
Ethics approval and consent to participate

The current research was established, according to the ethical guidelines of the Helsinki Declaration and was approved by the Human Ethics Committee of Yuhuangding Hospital on 12 January 2018 (NO. 2018149).

Written informed consent was obtained from all participants or legal guardian.

\section{Consent for publication}

Not applicable.

\section{Competing interests}

The authors declare that they have no competing interests.

\section{Author's Contributions}

Experimental design: LJS and GDW; Data collection: LJS, PZG and JWW; Data analysis: LJS, JWW, JW and XFW; Manuscript preparation: all. All authors reviewed the manuscript.

\section{Acknowledgements}

We appreciated that Dr Alicia Teijeira Crespo for modifying the manuscript.

\section{Availability of data and materials:}

The datasets used or analysed during the current study are available from the corresponding author on reasonable request.

\section{References}

1. Elmallah, R.K., et al., Pain Control in Total Knee Arthroplasty. J Knee Surg, 2018. 31(6): p. 504-513. DOI: 10.1055/s- 0037-1604152.

2. Zhao, X., et al., Efficacy of steroid addition to multimodal cocktail periarticular injection in total knee arthroplasty: a meta-analysis. J Orthop Surg Res, 2015. 10: p. 75. DOI: 10.1186/s13018-015-0214-8.

3. Dorr, L.D. and L. Chao, The emotional state of the patient after total hip and knee arthroplasty. Clin Orthop Relat Res, 2007. 463: p. 7-12.

4. Reuben, S.S. and A. Buvanendran, Preventing the development of chronic pain after orthopaedic surgery with preventive multimodal analgesic techniques. J Bone Joint Surg Am, 2007. 89(6): p. 1343-58. DOI: 10.2106/JBJS.F.00906

5. Davila Castrodad, I.M., et al., Rehabilitation protocols following total knee arthroplasty: a review of study designs and outcome measures. Ann Transl Med, 2019. 7(Suppl 7): p. S255. DOI: 
10.21037/atm.2019.08.15.

6. Bradshaw, P., S. Hariharan, and D. Chen, Does preoperative psychological status of patients affect postoperative pain? A prospective study from the Caribbean. Br J Pain, 2016. 10(2): p. 108-15. DOI: $10.1177 / 2049463716635680$.

7. Yue, C., et al., Effect of Topical Tranexamic Acid in Reducing Bleeding and Transfusions in TKA. Orthopedics, 2015. 38(5): p. 315-24. DOI: 10.3928/01477447-20150504-06.

8. Pattison, E., et al., Reduction in haemoglobin after knee joint surgery. Ann Rheum Dis, 1973. 32(6): p. 582-4.DOI: 10.1136/ard.32.6.582.

9. Fisher, D.A., et al., Looks good but feels bad: factors that contribute to poor results after total knee arthroplasty. J Arthroplasty, 2007. 22(6 Suppl 2): p. 39-42.DOI: 10.1016/j.arth.2007.04.011.

10. Adie, S., et al., Cryotherapy following total knee replacement. Cochrane Database Syst Rev, 2012(9): p. CD007911. DOI: 10.1002/14651858.CD007911.pub2.

11. Macedo, L.B., et al., Effect of burst TENS and conventional TENS combined with cryotherapy on pressure pain threshold: randomised, controlled, clinical trial. Physiotherapy, 2015. 101(2): p. 15560. DOI: 10.1016/j.physio.2014.07.004.

12. Block, J.E., Cold and compression in the management of musculoskeletal injuries and orthopedic operative procedures: a narrative review. Open Access J Sports Med, 2010. 1: p. 105-13. DOI: 10.2147/oajsm.s11102

13. Chai, X., et al., Efficacy of Additional Corticosteroid in a Multimodal Cocktail for Postoperative Analgesia Following Total Knee Arthroplasty: A Meta-Analysis of Randomized Controlled Trials. Pain Pract, 2019. 19(3): p. 316-327. DOI: 10.1111/papr.12740.

14. Mistry, J.B., et al., Rehabilitative Guidelines after Total Knee Arthroplasty: A Review. J Knee Surg, 2016. 29(3): p. 201-17. DOI: 10.1055/s-0036-1579670.

15. Xie, J., et al., Comparison of intravenous versus topical tranexamic acid in primary total hip and knee arthroplasty: An updated meta-analysis. Thromb Res, 2017. 153: p. 28-36. DOI: 10.1016/j.thromres.2017.03.009.

\section{Figures}



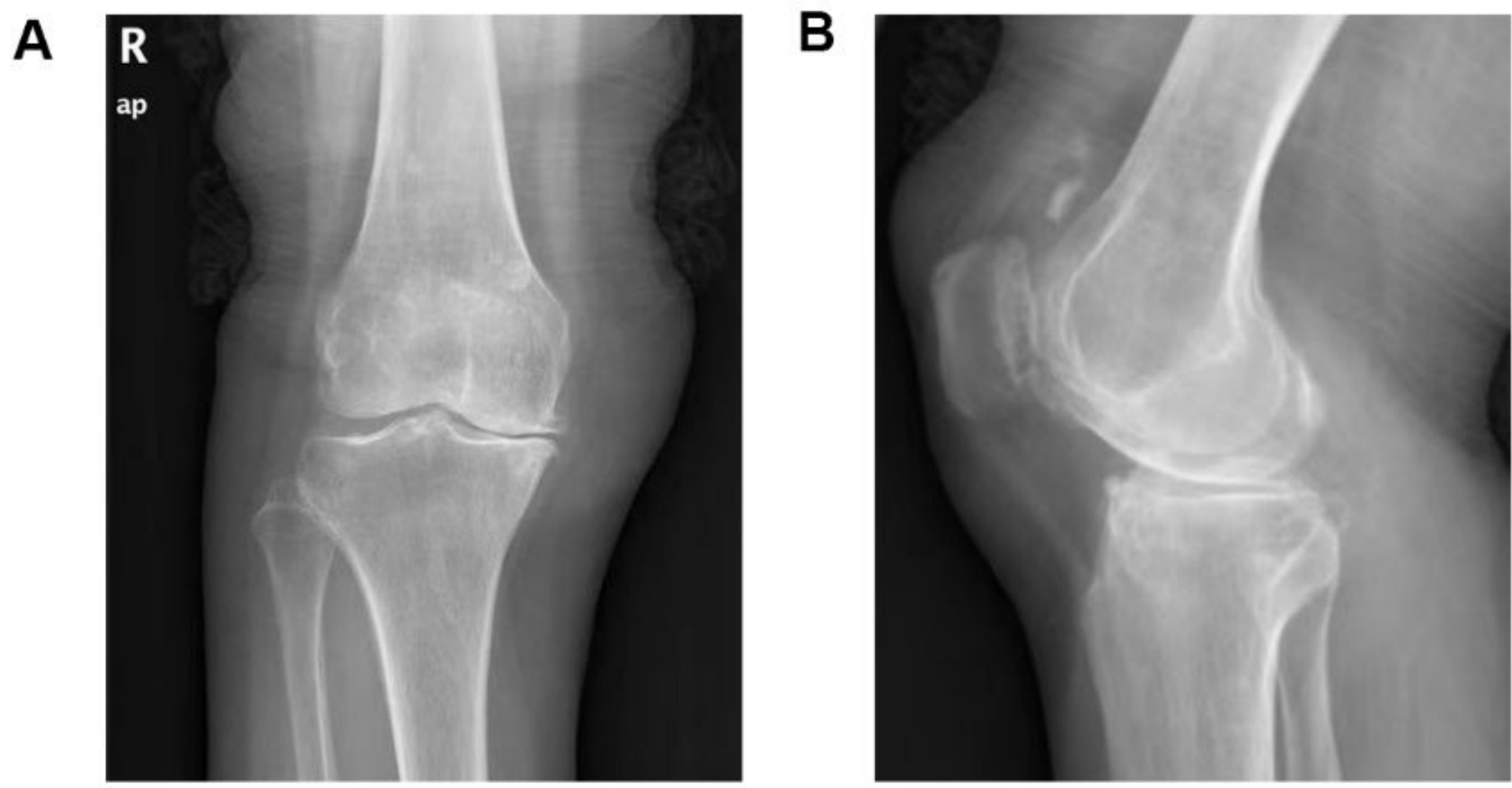

\section{Figure 1}

Preoperative X-ray of the knee. (A) X-ray was taken from the anterior and posterior ankle of the knee. (B) $X$-ray was taken from the lateral ankle of the knee.

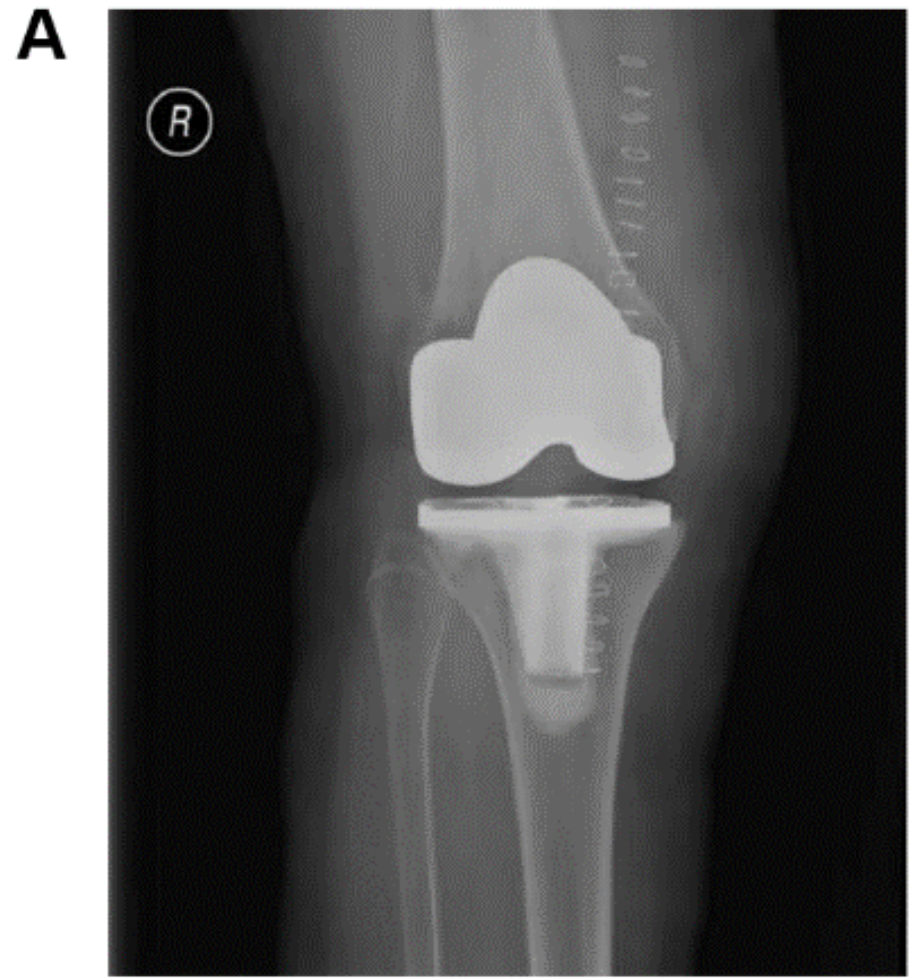

B

Figure 2 
Postoperative X-ray of the knee. (A) X-ray was taken from the anterior and posterior ankle of the knee. (B) $X$-ray was taken from the lateral ankle of the knee.

\section{Assessed for eligibility $(\mathrm{n}=70)$}

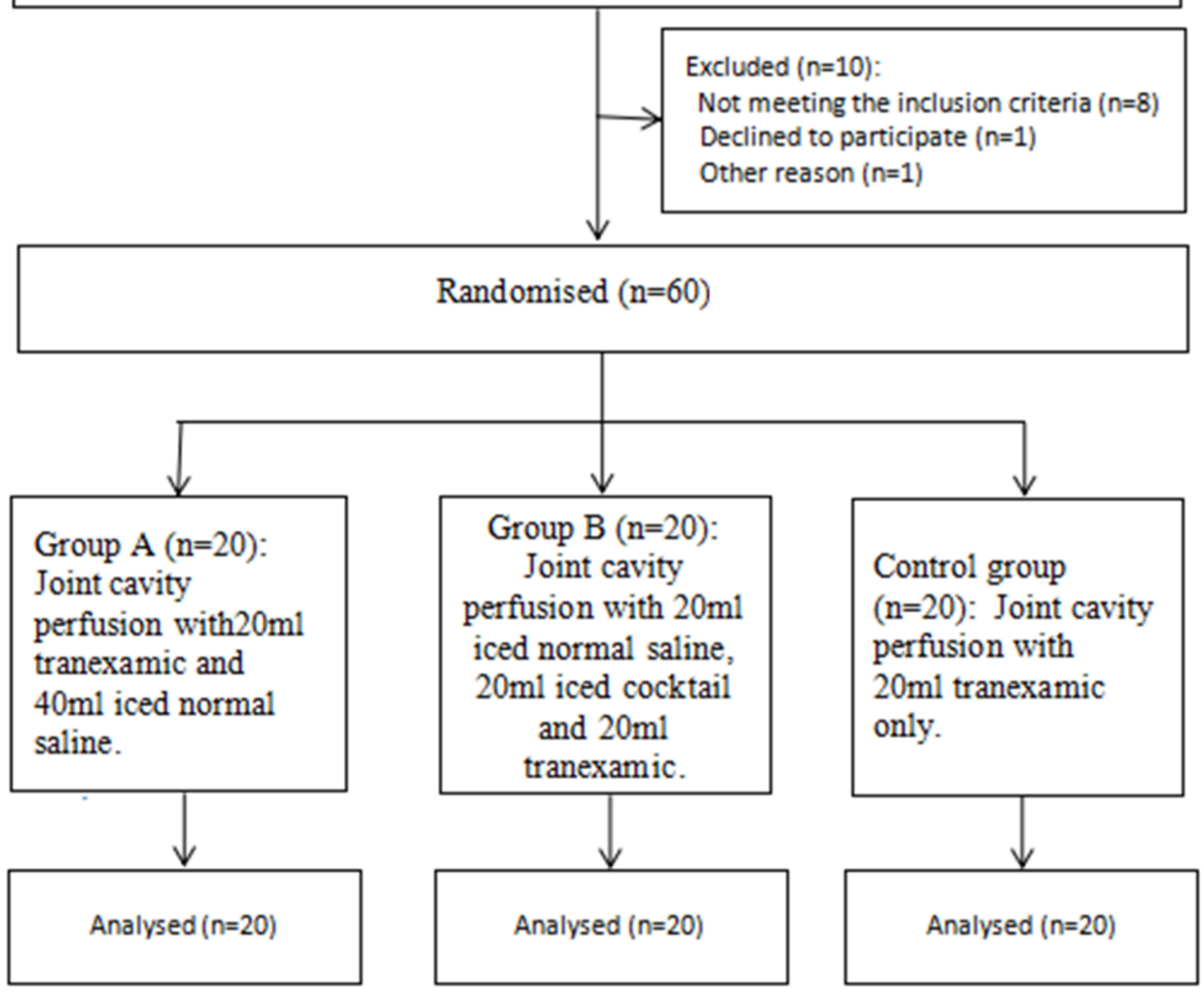

\section{Figure 3}

Flow chart of the present study through the phased of a randomised trial of three groups. 

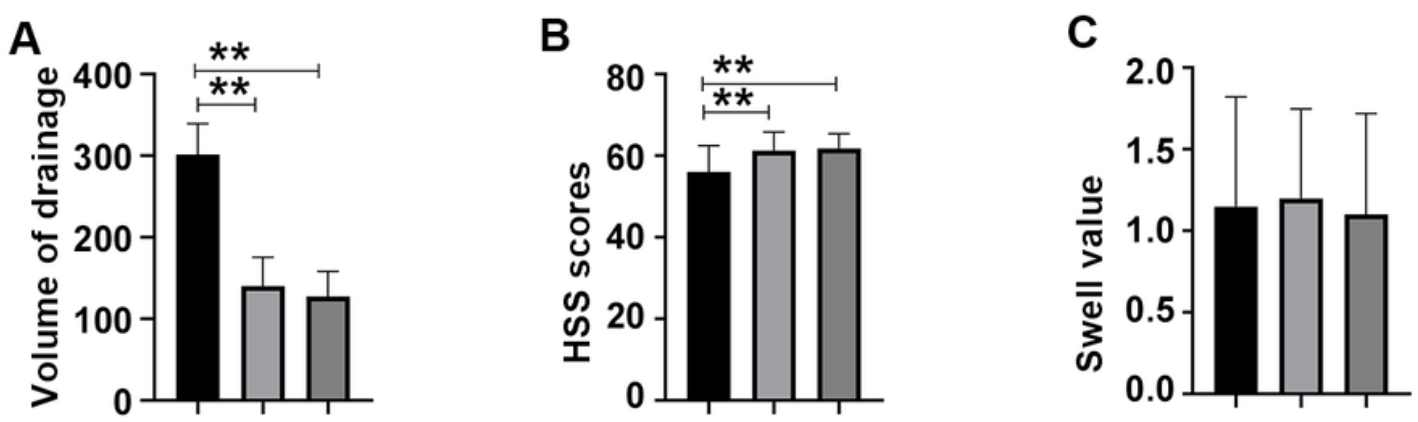

- Control group $(\mathrm{n}=\mathbf{2 0})$

口 Group A ( $\mathrm{n}=20)$

$\square$ Group B $(n=20)$
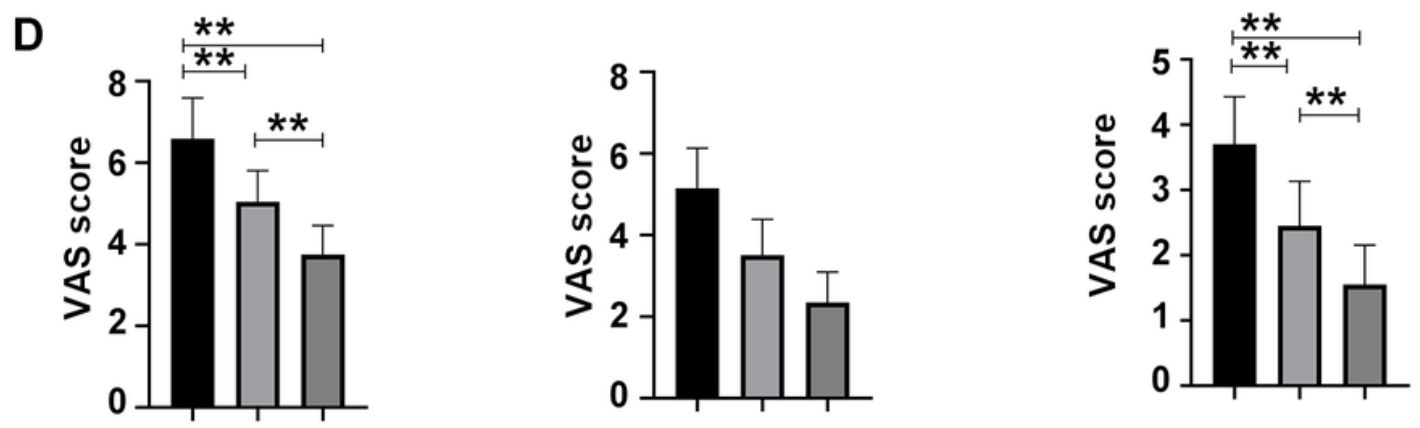

Figure 4

Altered postoperative observation indexes in three groups. (A) Histogram to present differential postoperative drainage volumes in three groups. (B) Altered postoperative HSS scores in three groups. (C) Swelling values after operation in three groups. (D) VAS scores in the groups were shown in the 24 hours, 48 hours and 72 hours after operation, respectively. ${ }^{\star *} P<0.01$. 